\title{
ULTRA-HIGH RESOLUTION IMAGING OF FACADES AND VERTICAL INFRASTRUCTURE BY CARBORNE SAR AND AIRBORNE CSAR
}

\author{
S. Palm ${ }^{1, *}$, R. Sommer ${ }^{1}$, A. Tessmann ${ }^{2}$, U. Stilla ${ }^{3}$ \\ ${ }^{1}$ Fraunhofer FHR, 53343 Wachtberg, Germany - (stephan.palm, rainer.sommer)@fhr.fraunhofer.de \\ ${ }^{2}$ Fraunhofer IAF, 79108 Freiburg, Germany - axel.tessmann@iaf.fraunhofer.de \\ ${ }^{3}$ Photogrammetry and Remote Sensing, Technical University of Munich (TUM), Germany - stilla@tum.de
}

ICWG II/III: Pattern Analysis in Remote Sensing

KEY WORDS: Ultra-high Resolution SAR, Carborne SAR, Airborne CSAR, Facade Mapping, Vertical Infrastructure Mapping, Mobile Radar Mapping

\begin{abstract}
:
In this paper we propose a strategy to focus ultra-high resolution single channel carborne SAR and airborne circular SAR (CSAR) data to image facades and vertical infrastructure. We illustrate the related theoretical background and the design of an optimal focusing geometry for carborne SAR applications while using backprojection focusing techniques. Of particular interest is thereby the determination of the minimum distance and orientation of the facade to the radar sensor. Potential image distortions due to a wrong choice of these parameters are illustrated. Effects on the final resolution of the data due to the rotation of the focusing geometry compared to typical airborne SAR are discussed. We validated the strategy by driving on conventional roads illuminating facades with an experimental mobile radar mapping (MRM) sensor operating at $300 \mathrm{GHz}$. We further present an adapted version of the proposed strategy to focus vertical infrastructure in CSAR data sets. By extracting the center coordinate and the principal orientation of an object from GiS data, the focusing plane is designed arbitrarily in the 3D space. For the CSAR data set, a radar sensor particularly designed for circular flight trajectories operating at $94 \mathrm{GHz}$ was evaluated. An electrical pylon was chosen as potential target. In both applications, the final images show a high level of detail. The combination of proposed strategy and radar sensor with very high bandwidth is capable of subcentimeter imaging of facades. The height, shape and dimensions of objects can be extracted directly from the image geometry at very high accuracy.
\end{abstract}

\section{INTRODUCTION}

Synthetic aperture radar imaging (SAR) is a well established remote sensing technology commonly applied in the fields of earth observation or site monitoring (Moreira et al., 2013). Radar as an active sensor can provide high-resolution images for many applications daylight- and weather independent. For imaging infrastructure at close ranges in ultra high resolution, mobile mapping with cameras and laser sensors is still state of the art technology, see (Paparoditis et al., 2012, Gehrung et al., 2017). Due to the miniaturization of radar sensors and a continuous increase in RF bandwidth in the last years, compact carborne mobile radar mapping (MRM) sensors become more attractive for these applications. Current applications which are investigated are topography mapping in Ku-band (Frey et al., 2018), automotive applications like parking lot detection (Feger et al., 2017) or the successful demonstration of road mapping on a subcentimeter resolution scale (Palm et al., 2018). High resolution imaging of vertical infrastructure is still a challenging field due to the side looking geometry of the sensor causing layover and multipath effects. Typical methods to overcome these challenges in airborne or satellite applications are SAR tomography and interferometry. Recently, authors in (Uemoto et al., 2018) presented a method for extraction and height estimation of vertical structures from X-band InSAR data. Tomographic SAR allows 3D imaging of point like scatteres and can further be used to analyze the vertical height and structure of vegetation layers with penetration of the sensor's radiation typically at lower frequencies (Reigber et al.,

* Corresponding author
2015). In this paper we propose a strategy to focus ultra high resolution SAR data of near vertical infrastructure by operating a carborne MRM sensor. As time domain backprojection techniques need precise knowledge of the scene, the process of focusing the SAR data is thereby performed directly in the adapted, vertical image geometry. This simultaneously allows the extraction of height information, form, shape and dimensions of objects from the image geometry in very high detail. Besides the imaging with optical or laser sensors, the surface roughness and backscattering behaviour of objects can be analyzed in a different waveband. To achieve ultra high resolution, radar sensors in the $\mathrm{mm}$ wavelength domain are very promising as they can generate high RF bandwidths. Therefore, we conducted the carborne experiments with an FMCW radar sensor operating at $300 \mathrm{GHz}$, which corresponds to $1 \mathrm{~mm}$ wavelength. To our knowledge, carborne mobile radar mapping experiments to measure close vertical infrastructure were not investigated, yet. By further adapting the presented method for airborne applications, we were able to successfully image vertical objects in airborne circular SAR (CSAR) data. By extracting the center coordinate and the principal, main orientation of the potential object from GiS data, we were able to design an optimal focusing geometry which can be placed arbitrarily in the $3 \mathrm{D}$ domain. From the full aspect CSAR geometry we are then able to select the relevant data from the $360^{\circ}$ spotlighted data set with optimal view to the target. To experimentally validate this technique, we used a radar sensor particularly designed for circular flight trajectories operating at $94 \mathrm{GHz}$. The focused data for both applications can at last be transferred to conventional 3D point cloud software. 


\section{METHOD}

\subsection{Imaging of facades by carborne SAR}

In airborne or spaceborne stripmap SAR known from the literature, the sensor typically points down illuminating the scene with a fixed depression angle $\delta$ between usually $30^{\circ}-$ $45^{\circ}$. The same geometry was applied to successfully map close infrastructure or streets in subcentimeter resolution with carborne sensors, see (Palm et al., 2018). By a modification of the sensor's antenna look direction, the mapping of vertical structures such as housing facades or trees becomes feasible in carborne SAR applications. Therefore, we modified the depression angle by $\approx 60^{\circ}-70^{\circ}$ resulting in an upward antenna look direction with an elevation angle of $\theta \approx 30^{\circ}-40^{\circ}$, depending on the actual objects. This allows the illumination of vertical structures at relatively close distances by driving the sensor platform on conventional roads in side looking geometry. With no GiS or DSM information at hand, the correct estimation of the distance from sensor to the surface which is to be imaged is more complex than in the airborne or street mapping case. In these both scenarios the mean sensor height above ground is usually a reasonable first estimation, which can be evaluated by GPS/INS data. The distance to facades, however, can be difficult to predict and may be subject to frequent fluctuations while moving the sensor on the road. As we further do not have detailed information of the shape or orientation of the buildings which are illuminated, the use of flat, vertical planes to focus the radar data is implemented as a forward strategy. Fig. 1 indicates the described geometry of facade mapping for a typical urban scenario.

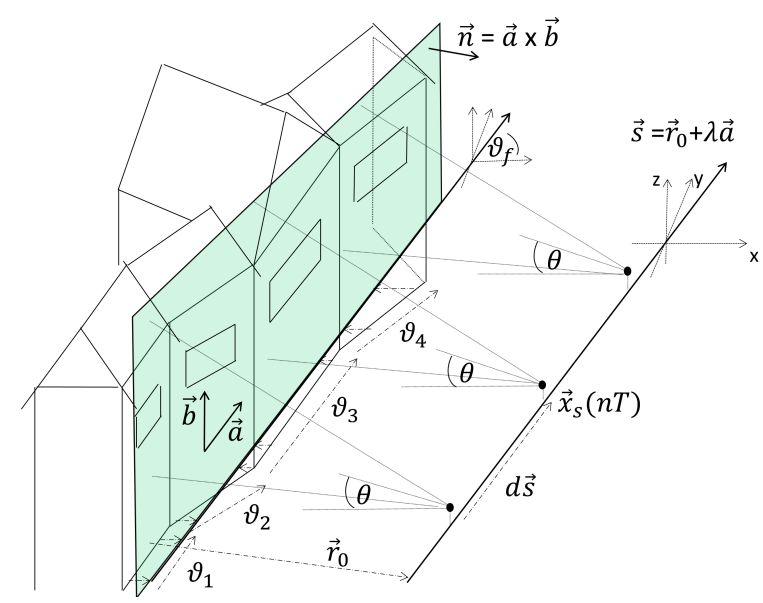

Figure 1. Representation of facade focusing geometry by carborne upward looking stripmap SAR. The focusing plane is indicated in green.

Here, we assume vertically built objects and facades. Their mean orientation angle $\vartheta_{f} \approx \frac{\sum_{i=1}^{n} \vartheta_{i}}{n}$ is estimated to be parallel to the street and thus parallel to the trajectory of the sensor $\vec{s}$ indicated as

$$
\vec{s}=\vec{r}_{0}+\lambda \vec{a} .
$$

with $\vec{r}_{0}$ the start of the sensor's trajectory, $\vec{a}$ the mean directional vector of the sensor's trajectory $\vec{s}$ and $\lambda \in \mathbb{R}>0$. The sensor's actual position $\vec{x}_{s}(n T)$ and movement can be derived by an integration or discrete sum of the sensor velocity components

$$
\begin{gathered}
\vec{x}_{s}(n T)=\vec{r}_{0}+\int_{0}^{n T} \vec{v}(t) \cdot t d t \approx \vec{r}_{0}+\sum_{m=1}^{m=n}\left(\begin{array}{c}
v_{e}(m T) \\
v_{n}(m T) \\
v_{d}(m T)
\end{array}\right) T, \\
m, n \in \mathbb{N}, 0<m \leq n
\end{gathered}
$$

with $n \in \mathbb{N}$ as the sweep index, $T$ the sweep duration which is the inverse of the sweep repetition frequency, $t$ the fast time and the corresponding sensor velocities $v_{e}(m T)$ (easting), $v_{n}(m T)$ (northing) and $v_{d}(m T)$ (down). The assumption of a mean orientation of the facades is reasonable, since houses are often aligned to the street direction. The focusing plane can then be described by a normal vector $\vec{n}$ perpendicular to the trajectory. In contrast to the flat plane model with $\vec{n}_{a}=$ $(0,0,1)^{\prime}$ in airborne case, however, the facade plane can be defined arbitrarily in space, depending on the sensor's trajectory and the estimated orientation of the facade. In a typical urban scenario, the green focusing plane in the cartesian coordinate system of Fig. 1 can then be described as

$$
\vec{E}=\vec{x}_{0}+\mu_{1} \vec{a}+\mu_{2} \vec{b}, \mu_{i} \in \mathbb{R}
$$

with $\vec{x}_{0}=\overrightarrow{0}$, corresponding to a zero crossing of the plane to the coordinate system and $\vec{b}=(0,0,1)^{\prime}$, assuming a vertical facade structure, which is reasonable for most man made buildings. With the derivation of the normal vector $\vec{n}$ of the image plane as

$$
\vec{n}=\vec{a} \times \vec{b}
$$

we end up in a right-handed trihedron. In comparison to the typical down looking stripmap SAR the entire focusing geometry is rotated by $90^{\circ}$ as indicated in Fig. 2. If the antenna direction is thus not rotated by $90^{\circ}$ this will affect the final projected resolution in slant range direction. With $\delta_{x}$ as the slant range resolution and $\delta_{y}$ the azimuth resolution it holds

$$
\begin{aligned}
\delta_{x, g} & =\frac{c_{0}}{2 B \cdot \cos (\delta)}=\frac{c_{0}}{2 B \cdot \cos (\theta)} \\
\delta_{y} & =\frac{\lambda}{4 \tan \left(\frac{\varphi}{2}\right)},
\end{aligned}
$$

where $\delta_{x, g}$ is the ground range resolution, B the bandwidth, $\varphi$ the main lobe coherent aspect integration interval, $\delta$ the depression angle and $\theta$ the elevation angle. In the case of vertical focusing we can derive

$$
\begin{aligned}
\delta_{x, v} & =\frac{c_{0}}{2 B \cdot \cos \left(\frac{\pi}{2}-\theta\right)}=\frac{c_{0}}{2 B \cdot \sin (\theta)} \\
\delta_{y} & =\frac{\lambda}{4 \tan \left(\frac{\varphi}{2}\right)}
\end{aligned}
$$

with $\delta_{x, v}$ as the 'range' resolution projected on the vertical axis which corresponds to height. Assuming the antenna's radiation direction is also rotated by $90^{\circ}$ this has no effect on the system's achievable resolution capacity of scatterers in range and azimuth direction. However, depending on the height of the building and distance to the street, the elevation angle $\theta$ can be subject to frequent fluctuations. In typical vertical mobile radar mapping scenarios the elevation of the antennas will be flatter which results in a slightly reduced resolution in vertical height compared to ground range. This is further visualized in Fig. 2. With $r_{c}$ as the smallest, perpendicular distance from the start of the sensor's trajectory to the facade, the start position $\vec{r}_{0}$ 


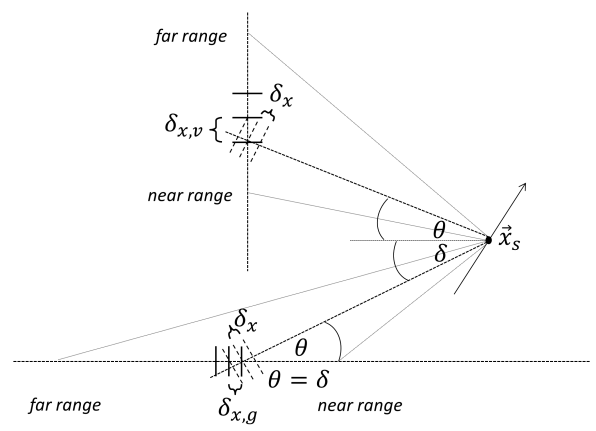

Figure 2. Comparison of down looking and upward looking stripmap SAR geometry with respect to projected resolution. The focusing geometry is rotated by $90^{\circ}$.

of the trajectory can be selected to $\vec{r}_{0}=\overrightarrow{0} \pm \vec{n} \cdot r_{c}$ depending on which side (left or right) the sensor looks.

With the accuracy of the used GPS/IMU, the cartesian coordinate system is then defined with $\overrightarrow{\mathbf{x}}$ corresponding to the geographical easting, $\overrightarrow{\mathbf{y}}$ to northing and $\overrightarrow{\mathbf{z}}$ to the height. This allows the application of backprojection processing routines for the presented methodology and the subsequent transfer of the focused data to conventional point cloud software.

With an elevation angle $\theta$ the facade is then constantly illuminated by the electromagnetic waves of the radar. In particular problematic is the determination of the smallest distance $r_{c}$ from the sensor to the facade at the start of the trajectory. Again, if there is no prior information by the use of a second sensor or GiS data, the distance $r_{c}$ has to be estimated adequately. At small elevation angles, $r_{c}$ can further be estimated based on the first echoes of the radar. In stripmap mode, wrong assumptions will not lead to a defocusing process of the final image, but to a misplacement (foreshortening) of the focused scatterers in the final image domain, see (Duersch , Long, 2015). However, as the movement of the sensor platform in practice will not always be perfectly linear, this additionally leads to a certain degree of blurring and loss of peak signal power in the processing. This is especially the case when considering very high bandwidths as discussed in this paper. Therefore, assuming a fixed distance $r_{c}$ at the start of the trajectory becomes problematic when considering the focusing of longer trajectories since the distance from house to house can be different.

In many cases the orientation of the individual facades $\vartheta_{i}, i \in \mathbb{N}$ will not be consistent to the derived plane's orientation $\vartheta_{f}$ (green image plane), as indicated in Fig. 1. If the angular difference $d \vartheta_{i}=\vartheta_{f}-\vartheta_{i}$ is small, the SAR focusing process will still generate sharply focused details, but the facades will be displayed visually slightly distorted. Similar to an optical image, with $d \vartheta \neq 0$, the facades and windows will either be displayed to come out of the image plane or move into it. The following applies: For $d \vartheta_{i}<0$ the facade moves away from the sensor, for $d \vartheta_{i}>0$ the facade moves towards the sensor. This information can be further exploited to extract 3D information or to adapt autofocus routines but these methods are not discussed in this paper so far. A limitation of the described method is that the trajectory of the platform $\vec{s}$ should be relatively linear and the minimal distance $r_{c}$ from radar to the facades relatively constant. If the road is too curvy, this would cause the sensor to move into the focusing plane or too far away. However, this can be avoided if we extend the focusing geometry by adapted planes. Depending on the respective lay-out of the road, the actual minimal distance $r_{c}$ can then be adapted by the use of the GPS/IMU data. The individual vertical focusing planes can then be adapted down to the level of each overlapping subaperture resulting in a robust processing chain for longer distances. Due to spatial decorrelation in this high frequency domain, the SAR images with subcentimeter resolution appear noisy when processed in single look mode. We therefore use a multilook processing, see (Cumming, Wong, 2005), to noncoherently superimpose slightly changing aspect information in each pixel. This is unproblematic as we do not need the full achievable bandwidth in azimuth in order to process the same level of resolution in range as in azimuth. An overlapping multilook factor of 2 or 3 is implemented. This increases the contrast of the final images and reduces speckle behaviour.

\section{Defining a suitable facade orientation by GiS information}

The presented method works robustly for most data sets based on the assumption of a parallel facade orientated to the street. If available, a second option to assume the average facade orientation $\vartheta_{f}$ of single houses or of a whole row of houses is the use of GiS data. Nowadays, there are many public and free software products which are adequate to extract these information. Particularly suitable are georeferenced aerial or satellite images (orthophotos) where individual facade orientations can immediately be extracted. This can significantly improve the accuracy of the focusing plane and thereby reduce possible distortions in the focused SAR image. Even longer row of houses or sections with slightly winding roads can be imaged in better quality. The orientation angle $\vartheta_{f}$ is then evaluated as a further input parameter in the processing step, see section 3 .

\subsection{Processing vertical infrastructure in airborne CSAR}

We can further adopt the developed method of vertical mapping for the processing of airborne SAR or in particular for CSAR. Image distortions which occur by focusing vertical objects can be reduced and the resolution can be increased. In CSAR, the sensor moves along a circular shaped trajectory over a defined, spotlighted target region with coordinates $R(\varphi)=$ ( $\left.R_{m} \cdot \cos (\varphi), R_{m} \cdot \sin (\varphi), h_{z}\right)$ with $R_{m}$ as the radius of the circle and $\varphi \in[0,2 \pi]$ as the azimuth angular variation or aspect, see Fig. 3. The continuously changing current flight direction is indicated with $\vartheta_{n T}$ (red arrow). The target's center coordinate $P_{m}$ is then illuminated from all possible aspect angles with a depression angle of $\delta_{n T}$. From (Ponce et al., 2014), the collected radar data of a specific target region $\vec{x}$ and transmitted pulse $P_{\omega}(\omega)$ can then be described in the spatial frequency domain as

$$
S(\vec{k}, \varphi)=P_{\omega}(\omega) \int \alpha\left(\vec{r}_{g}\right) \cdot e^{-j 2 k \cdot\left\|\vec{r}_{g}-\vec{x}_{s}(\varphi)\right\|} d \vec{r}
$$

with $\alpha\left(\vec{r}_{g}\right)$ as the complex reflectivity of the imaging grid with $\vec{r}_{g}=\left(r_{x}, r_{y}, r_{z}\right)$, the sensor's position $\vec{x}_{s}$ and $k=\|\vec{k}\|=\frac{2 \pi}{\lambda(\omega)}$ as the wavenumber with $\omega$ as the fast-time angular frequency. The calculation of the unknown complex reflectivity of the scatterer position $\vec{x} \in \mathbb{R}$ can then be transferred to a coherent two dimensional integration of the projected spatial frequencies

$$
\alpha\left(\vec{r}_{g}\right)=\int_{k} \int_{\varphi} k \cdot e^{-j 2 k \cdot\left\|\vec{x}-\vec{x}_{s}(\varphi)\right\|} \cdot e^{j 2 k \cdot\left\|\vec{r}_{g}-\vec{x}_{s}(\varphi)\right\|} d \varphi \cdot d k .
$$


The degree of resolution is then determined by the ability of a coherent integration of a preferably large aspect interval $\varphi$. From (Ishimaru et al., 1998), assuming a $360^{\circ}$ integration, the resolution in both dimensions azimuth and range can be derived to

$$
\delta_{x}=\delta_{y}=\frac{4.8 \cdot \lambda}{4 \pi \cdot \cos \delta}
$$

which corresponds to a theoretical resolution of $<0.002 \mathrm{~m}$ in the used frequency domain of $94 \mathrm{GHz}$ and a planar target. In

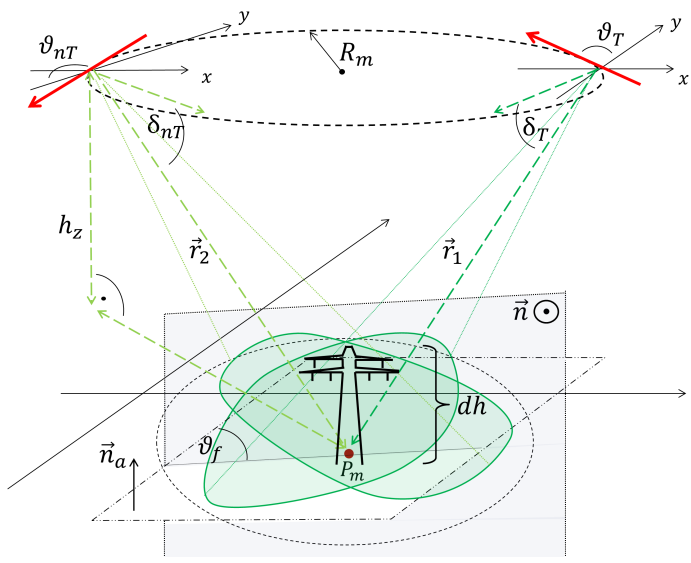

Figure 3. Representation of two different views by overlapping beams on a vertical target in CSAR geometry.

The design of the vertical focusing plane with normal vector $\vec{n}$ is indicated in blue.

practice, however, in this high frequency domain the resolution derived in literature is not feasible due to many limitations (planar, isotropic scatterer, limitations of GPS/IMU device, visibility of target...).

In the presented methodology of CSAR processing on vertical focusing planes, the target's center position $P_{m}$ and the mean orientation $\vartheta_{f}$ of the object are to be defined as input parameters. Each pixel $\left(x_{g}, y_{g}\right)$ on the specified 2D image grid $\vec{E}=\vec{x} \cdot \vec{n}=0$ with constant pixel spacing $\delta_{p}$ is mapped to a potential scatterer position $\vec{x}_{p}$ in the $3 \mathrm{D}$ cartesian coordinate system as

$$
\begin{aligned}
& x_{p}=x_{0}+\sin \left(\vartheta_{f}\right) \cdot\left(x_{g}-\frac{x_{\text {max }}}{2}\right) \cdot \delta_{p} \\
& y_{p}=y_{0}+\cos \left(\vartheta_{f}\right) \cdot\left(x_{g}-\frac{x_{\text {max }}}{2}\right) \cdot \delta_{p} \\
& z_{p}=z_{0}+y_{g} \cdot \delta_{p}, \quad y_{g} \cdot \delta_{p} \leq d_{h}
\end{aligned}
$$

with $x_{0}, y_{0}, z_{0}$ the coordinate of the center position $P_{m}=$ (Lat, Lon, Height $) \rightarrow\left(x_{0}, y_{0}, z_{0}\right)$ and $d_{l}$ as the length and $d_{h}$ the height of the defined focusing plane, see Fig. 4. $x_{\max }$ corresponds to $\frac{d_{l}}{\delta_{p}}$.

The full $360^{\circ}$ trajectory is further divided in each overlapping subapertures of a constant aspect integration interval of usually $0.6^{\circ}-8^{\circ}$ depending on the potential target and processing mode. Images with large integration intervals in particular require an exact evaluation of the input parameters $P_{m}$ and $\vartheta_{f}$ as the focusing in very high resolution mode requires exact knowledge of the scatterer's 3D position. This is different to linear SAR processing where non planar targets are only displaced in the image geometry (foreshortening...) but are in addition not affected by blurring and peak signal loss, see (Cantalloube, 2018). Given the vertical plane model of Fig. 3,

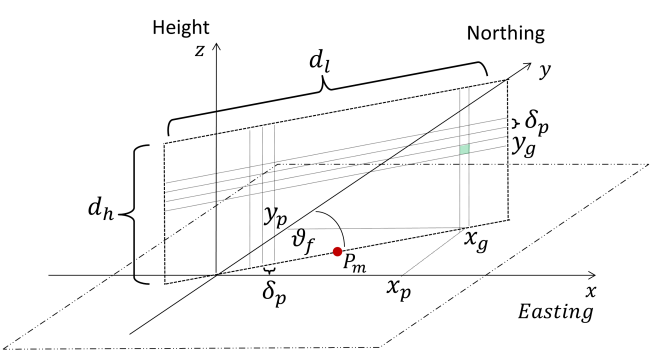

Figure 4. Design of vertical focusing plane in CSAR geometry. Input parameters are $P_{m}$ and orientation $\vartheta_{f}$.

for each circular subaperture we define the $3 \mathrm{~dB}$ main lobe of the beam on the focusing plane. With $\vec{l}$ as the line of sight vector of the antenna we can compute the intersection point $\vec{p}_{i}$ with the vertical plane as

$$
\vec{p}_{i}(n T)=\vec{p}_{s}(n T)+\frac{-\vec{n}^{\prime} \cdot \vec{p}_{s}(n T)}{\vec{n}^{\prime} \cdot \vec{l}(n T)} \cdot \vec{l}(n T) .
$$

With $\vec{p}(n T)$ and the specific $3 \mathrm{~dB}$ beamwidth the illuminated area $\mathbf{X}_{0}(n T)$ on the vertical plane can be defined. Based on this geometry the time domain backprojection focusing process is established.

\section{EXPERIMENTS}

\subsection{Carborne facade mapping campaign}

The radar sensor which was investigated for the carborne facade mapping applications is an experimental, compact frequency modulated continuous wave (FMCW) sensor, see (Palm et al., 2018). It operates at a center frequency of $300 \mathrm{GHz}$ with up to $50 \mathrm{GHz}$ bandwidth (Tessmann et al., 2014). The radar front-end and back-end is installed in a van which can be used on conventional roads.

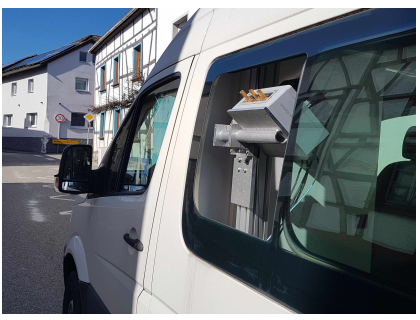

(a)

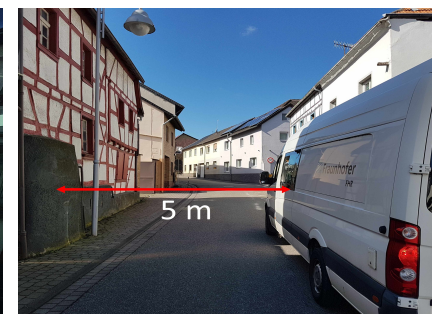

(b)
Figure 5. Carborne SAR experiments. (a) Antennas and front-end behind the open window. (b) Sensor platform and measuring distance.

Transmit and receive antennas are positioned behind the open window of the platform in side looking configuration (upwards direction), see Fig. 5a. The GPS antenna is placed on top of the van, the sensor's back-end and IMU is installed in the interior. We conducted experimental campaigns on conventional roads in several towns illuminating the facades of row of houses with a typical elevation angle of $30^{\circ}-35^{\circ}$. While collecting the data, the typical length of a trajectory was $50 \mathrm{~m}$ up to a few hundred meters, the distance to the facades was between 5 to $15 \mathrm{~m}$, see Fig. 5b. With sweep repetition frequencies (SRF) 
up to $10 \mathrm{kHZ}$ and a new developed digital signal converter the platform's velocity can reach up to $40 \mathrm{~km} / \mathrm{h}$ (for full bandwidth processing). The parameters of the sensor and the geometry for processing are further specified in table 1. Extracting the facade orientation of a row of houses which were illuminated in the campaign is done in GoogleEarth, see Fig. 7a.

Table 1. Carborne sensor and processing parameters

\begin{tabular}{l|l}
\hline Transmission Mode & FMCW \\
Frequency & $300 \mathrm{GHz}$ \\
Bandwidth & $30-50 \mathrm{GHz}$ \\
Sensor Dimensions & $20 \times 10 \times 10 \mathrm{~cm}$ \\
Weight SAR System & $5 \mathrm{~kg}$ \\
Power Consumption & $5 \mathrm{~V} \mathrm{/} 3 \mathrm{~W}$ \\
Output Power & $5 \mathrm{dBm}$ \\
GPS/INS System & IGI AEROControl \\
\hline Maximum Measuring Distance & $25 \mathrm{~m}$ \\
Measuring Depth & $12 \mathrm{~m}$ \\
Velocity of Van & up to $40 \mathrm{~km} / \mathrm{h}$ \\
Processed Bandwidth & $30 \mathrm{GHz}$ \\
Processed Resolution & $5 \mathrm{~mm}$ \\
\hline
\end{tabular}

\subsection{Airborne CSAR campaign}

The airborne sensor which was investigated for the CSAR campaign is an experimental, compact W-band FMCW sensor operating at $94 \mathrm{GHz}$ as available transmit power is far not sufficient to operate a $300 \mathrm{GHz}$ sensor in airborne mode. The sensor was particularly designed for the operation on circular trajectories on small airplanes. Therefore, the antennas of the front-end are integrated in a mechanical mount and a real-time interface was developed to communicate between mount and GPS/IMU device to ensure real-time beam steering capability, see (Palm et al., 2019).

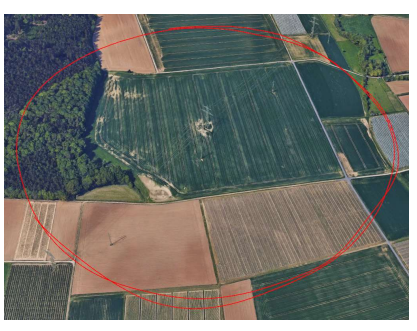

(a)

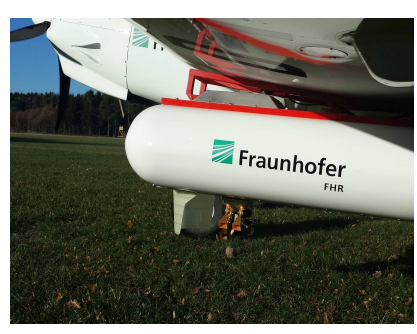

(b)
Figure 6. Airborne CSAR campaign. (a) GoogleEarth representation of circular flight trajectory with pylon in center. (b) W-band sensor below the airplane's wing.

This guarantees a $360^{\circ}$ view on the target region even in difficult flight conditions (windy conditions, small radius of circular trajectory...). The parameters of the sensor and the focusing geometry are listed in table 2. Fig. 6 shows the sensor and the trajectory of the platform at a height of $350 \mathrm{~m}$. We choose a $55 \mathrm{~m}$ high electrical pylon (height derived from GoogleEarth) as a potential target in the center of the beam. The mean roll angle of the airplane in the circle is about $8^{\circ}$ and thus the whole radar's depression angle is $40^{\circ}$. Again, the extraction of the center coordinate $P_{m}$ and the main orientation angle $\vartheta_{f}$ of the pylon is done in GoogleEarth, see Fig. 7b. For the CSAR subaperture focusing, we choose an aspect integration of $2.5^{\circ}$ corresponding to a theoretical resolution of $15 \mathrm{~cm}$ in range (1 $\mathrm{GHz} \mathrm{RF}$ bandwidth) and $4 \mathrm{~cm}$ in azimuth. The frame rate of the final CSAR videos (viCSAR) is selected to $20 \mathrm{~Hz}$ with high overlapping factor.
Table 2. CSAR sensor and processing parameters

\begin{tabular}{l|l}
\hline Transmission Mode & FMCW \\
Center Frequency & $94 \mathrm{GHz}$ \\
Bandwidth & up to $10 \mathrm{GHz}$ \\
Resolution video CSAR & $15 \mathrm{~cm}$ x $12 \mathrm{~cm}$ \\
Weight Front-End & $2.4 \mathrm{~kg}$ \\
Mount (Gimbal) & Somag CSM130 \\
Angular Steering Rate & $15^{\circ} / \mathrm{sec}$ \\
\hline Flight Height & $300 \mathrm{~m} \mathrm{AGL}$ \\
Flight Radius & $360 \mathrm{~m}$ \\
Flight Velocity & $35 \mathrm{~m} / \mathrm{s}$ \\
Azimuth Beamwidth & $10^{\circ}$ \\
Processed Aspect subaperture & $2.5^{\circ}$ \\
CSAR Video Frame Rate & $20 \mathrm{~Hz}$ \\
Pixel Spacing & $12 \mathrm{~cm}$ \\
\hline \multicolumn{2}{|l}{} \\
\hline
\end{tabular}

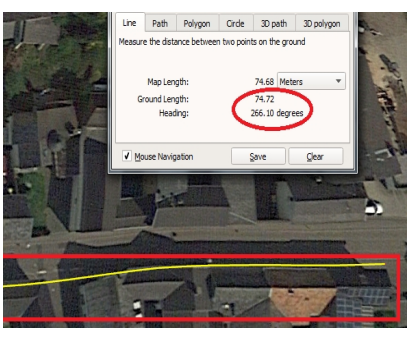

(a)

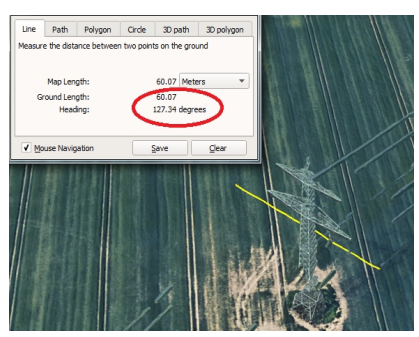

(b)
Figure 7. Extracting orientation $\vartheta_{f}$ of focusing plane from Google Earth. (a) Carborne facade mapping (b) Airborne infrastructure mapping.

\section{RESULTS}

The final SAR images in the facade mapping geometry were processed with a resolution of $5 \mathrm{~mm}$ which corresponds to 30 $\mathrm{GHz}$ bandwidth. Depending on the actual distance to the facade and the very high center frequency, this corresponds to a length of a synthetic aperture of typically below $0.5 \mathrm{~m}$. By the use of the proposed method the SAR images show very high focusing quality, a very high level of details, good contrast and are mapped with only minor distortions in the right geometry. The $\mathrm{Y}$ axis of the SAR images corresponds to the vertical height of the focusing plane. This allows to extract the actual height and width of facade elements like windows, masonry or rain pipes directly from the SAR images, if a correct orientation $\vartheta$ and distance $r_{c}$ is derived. In Fig. 8 the sequence of focusing the facade is presented. The whole facade is illustrated in Fig. 9a. The sampled real valued radar raw data are shown in Fig. 8a. In FMCW systems working with dechirp-on-receive, the image is formed by a superposition of sinusoidal functions in range, each corresponding to a single point target. After the range processing step, see Fig. $8 \mathrm{~b}$, strong targets, such as the top edge of the window appear in the typical parabolic form (range migration). The image itself remains barely interpretable. After azimuth compression, see Fig. 8c, fine details can easily be interpreted and the dimensions of potential targets can be measured in the image geometry. The height $\delta H$ and width $\delta L$ are indicated in the image in red.

In Fig. 9a the first floor of the full facade of the half-timbered house is presented in a resolution of $5 \mathrm{~mm}$. Due to the fact that the orientation of the facade and the orientation of the image plane are slightly shifted $(d \vartheta<0)$, the facade moves slowly into the image plane. This can be observed in particular on 


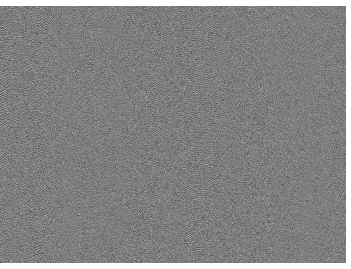

(a)

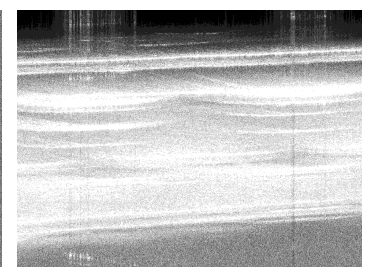

(b)

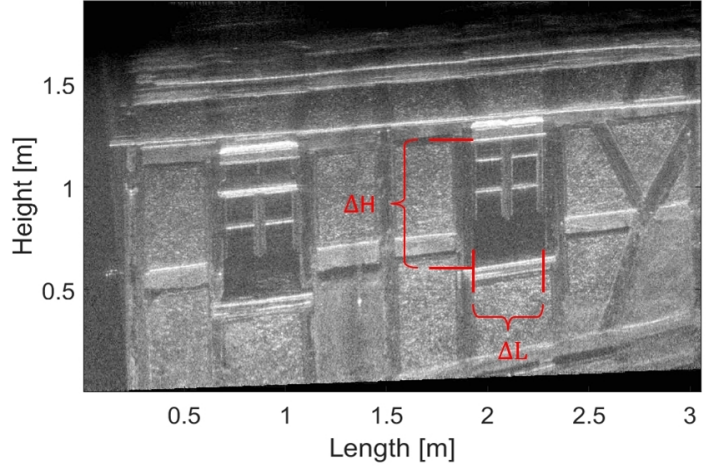

(c)

Figure 8. Sequence of facade focusing. (a) Collected real valued raw data. (b) Range compressed data. (c) Azimuth compressed facade image in vertical image geometry.

the course of the windows which are not exactly horizontal. The high level of details is clearly observable. The square framed timbered work of the facade can nicely be identified due to the different backscattering characteristic compared to the rough wall plaster. It can be observed that the wooden beams are slightly offset from the masonry and the scattering further reveals different workmanship of the masonry. The glass of the windows appears dark as the transmitted radar wave is reflected away (mirror effect). Due to the oblique view from below, window benches cause shadowing while we observe multipath effects and strong echoes on top of the windows. The strong horizontal echo from the top of the building is due to the rain pipe which can also be seen on the right side of the building. Similar effects can be observed in Fig. 9b, which shows two adjoining houses connected with an archway. Again, the shape and dimensions of the windows and their double bounce effects are clearly visible. The wall plaster of the right building was much smoother which leads to a very homogenous backscattering behaviour in the SAR image. The course of the rain pipe on the left side of the building is observable and again the double bounce effects due to the direct view into the roof projection. On the right side of the left building a tree is standing in front of the facade. The branches and leaves seem to partially project into the focusing plane. In Fig. 9c the brick facade of two houses is presented. The houses are situated next to each other but show a slightly different orientation angle. Due to the fine resolution each brick stone can be counted or the fixations of the rain pipe. The height $\Delta H$ and width $\Delta L$ of the windows were further evaluated. We used a laser rangefinder to measure the dimensions of the 3 windows marked red in Fig. 9. With a pixel spacing in the SAR image of $2 \mathrm{~mm}$ we could then manually analyze the dimensions in the SAR image, see Fig. 8. Table 3 compares the results.

In Fig. 10, the results of the airborne CSAR campaign are presented. Again, the potential height of the pylon can be extracted from the $\mathrm{Y}$ axis, see Fig. 11. The processed aspect
Table 3. Comparision of window size $\Delta H \times \Delta L$ measured (center) and extracted from SAR image (right)

\begin{tabular}{l|l|l}
\hline window Fig. 9a & $1.09 \mathrm{~m} \times 0.78 \mathrm{~m}$ & $1.14 \mathrm{~m} \times 0.74 \mathrm{~m}$ \\
window Fig. 9b & $1.12 \mathrm{~m} \times 0.76 \mathrm{~m}$ & $1.16 \mathrm{~m} \times 0.78 \mathrm{~m}$ \\
window Fig. 9c & $1.06 \mathrm{~m} \times 1.98 \mathrm{~m}$ & $1.03 \mathrm{~m} \times 1.98 \mathrm{~m}$ \\
\hline
\end{tabular}

interval for each subaperture was set to $2.5^{\circ}$ which corresponds to a theoretical resolution of $15 \mathrm{~cm}$ in height and $4 \mathrm{~cm}$ in azimuth (length). From the full CSAR data set we extracted the relevant sectors which provide a frontal view on both sides and a full illumination of the pylon. From Fig. 6a these views are $30^{\circ}$ - $60^{\circ}$ (front) or $210^{\circ}-240^{\circ}$ (behind) (orientation in north-east). The final images are very promising and show the detailed structures of the pylon. The backscattering characteristic is thereby highly sensitive to aspect changes, as the many metallic structures of the pylons partially flash or diminish. As the extracted center coordinate $P_{m}$ seems not to be the exact center of the $3 \mathrm{D}$ pylon, there is a slight offset in the position of the pylon when regarding the two different aspect views in Fig. 10 (a-b) compared to Fig. 10 (c-d). The height of the pylon from the focused image was measured to $56.5 \mathrm{~m}$, see Fig. 11 compared with the height from GoogleEarth of $55 \mathrm{~m}$. Due to the low echo from the pylon's base the exact height is difficult to determine, but already relative accurate.

\section{CONCLUSION}

In this paper we present a strategy to focus and map vertical infrastructure in ultra high resolution SAR images. The method can be used in carborne or airborne scenarios and was experimentally evaluated in both applications. The results are very promising since the final images show a very high level of resolution, contrast and detail. We could experimentally validate that a subcentimeter resolution of facades in SAR images is feasible. Very fine details or objects on facades can be revealed at day and night while the backscattering characteristic may help to distinguish different materials. In addition to LiDAR or optical photos the examination of facades can be supplemented by a considerably longer wavelength. We found out that the $1 \mathrm{~mm}$ wavelength of the sensor is very sensitive to brick, plaster or timbered house facades. The additional input of a laser system would even be advantageous, because the obtained geometry information of the laser could immediately improve the processing of the radar data. Of course, mapping also the lower part of the facade would require a second downlooking sensor at higher position.

In the CSAR part, we demonstrated that very high vertical infrastructure can be mapped from possibly all aspect angles if the sensor has a free view on the potential target. In contrast to conventional SAR, the object is less distorted in the final image and the height of the object can be directly derived from the image geometry. A high accuracy is of course only obtainable, if the focusing plane is located at the right position on the same level with the center of the 3D object. Many vertical infrastructure objects will have a main orientation and thus only very few aspect views might be relevant. With CSAR methods, the right view can always be extracted from the data set in retrospect. This can allow even the advanced imaging of different parts of such objects with focusing planes placed arbitrarily in the 3D space. The geometry would then be adapted to the size of individual objects which may only be evaluated from a certain aspect angle. 


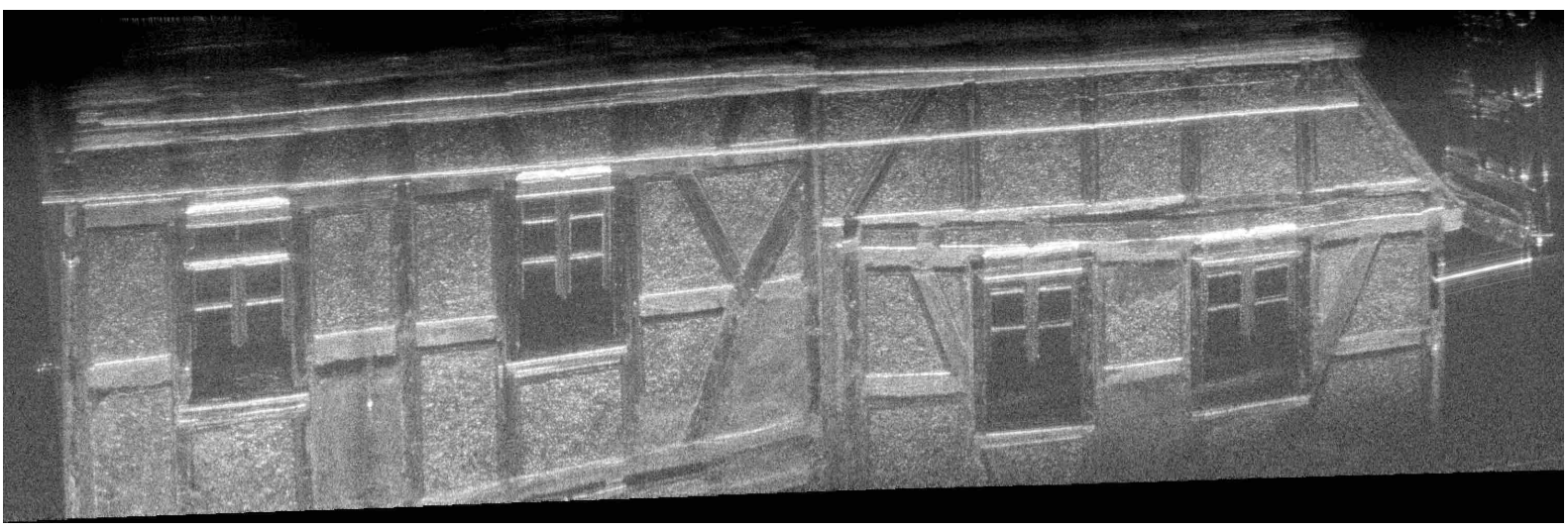

(a)

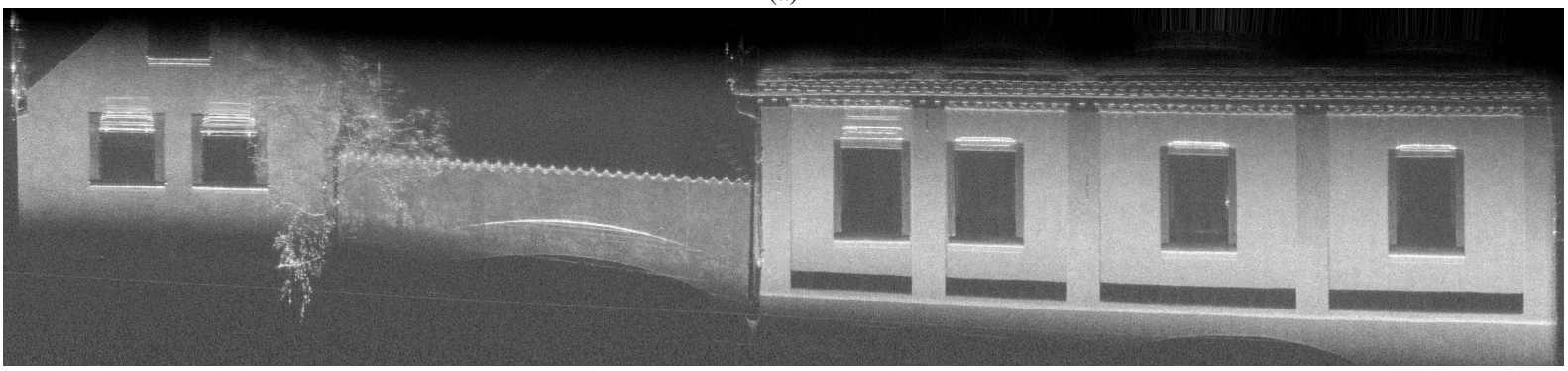

(b)

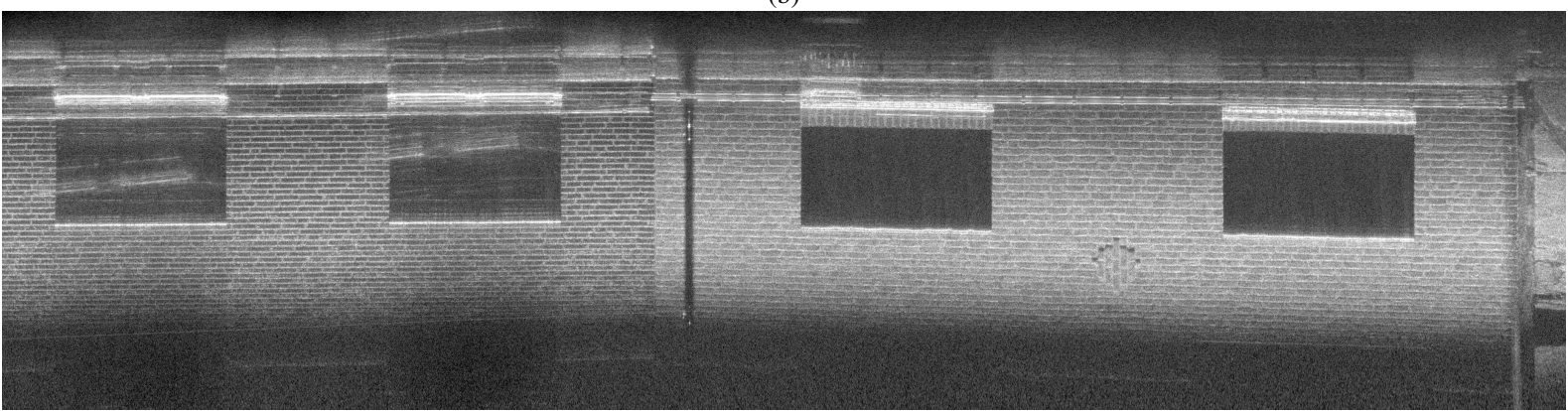

(c)

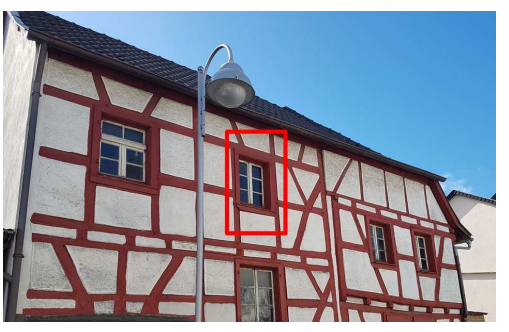

(d)

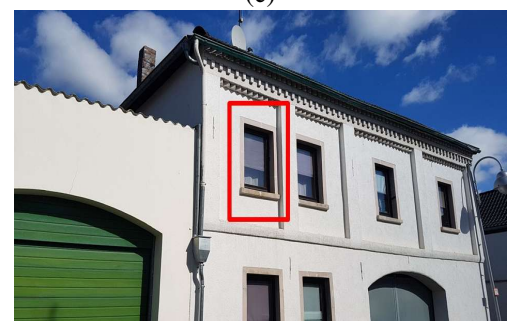

(e)

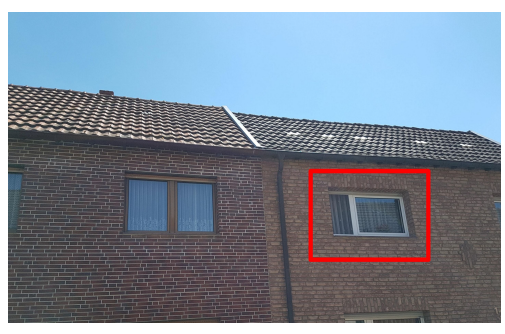

(f)

Figure 9. Carborne $300 \mathrm{GHz}$ SAR images of facades with $5 \mathrm{~mm}$ resolution. (a) First floor of half-timbered house. The orientation of the facade of the building is slightly offset from focusing plane. Thus, the windows and rain pipe seem to

move into the image plane. A very high level of details can be observed. The backscattering characteristic reveals

different materials. (b) First floor of two adjoining houses with more homogenous wall plaster connected with an archway. A tree is standing in front of the left facade. (c) Brick facade of two adjoining houses with slightly different orientation. (d) Photograph of half-timbered house. (e) Photograph of 2 adjoining houses. (f) Photograph of brick facade.

\section{REFERENCES}

Cantalloube, H. M., 2018. Circular SAR imaging of not planar targets. Limitations of the height from focus paradigm. IEEE International Geoscience and Remote Sensing Symposium, 2018. IGARSS 2018, 3671-3674.

Cumming, Ian G., Wong, Frank H., 2005. Digital Processing of Synthetic Aperture Radar Data: Algorithms and Implementation. Artech House Inc., Boston, London.
Duersch, Michael I, Long, David G, 2015. Analysis of time domain back projection for stripmap SAR. International Journal of Remote Sensing, 36, 2010-2036.

Feger, R., Haderer, A., Stelzer, A., 2017. Experimental verification of a $77-\mathrm{GHz}$ synthetic aperture radar system for automotive applications. 2017 IEEE MTT-S International Conference on Microwaves for Intelligent Mobility, 111-114.

Frey, Othmar, L. Werner, Charles, Hajnsek, I, Coscione, 


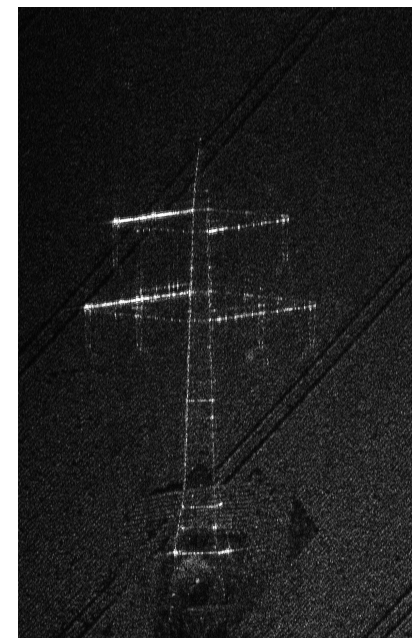

(a)

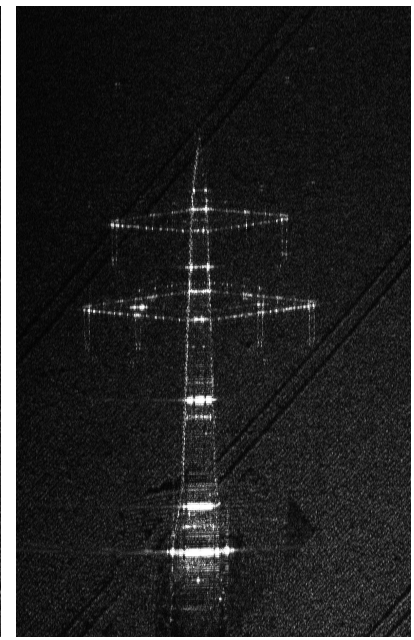

(b)

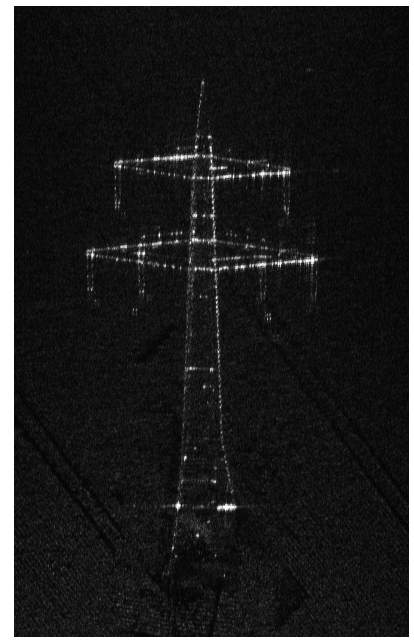

(c)

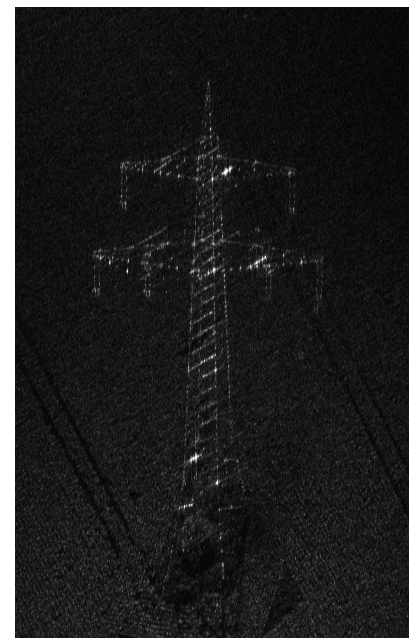

(d)

Figure 10. CSAR subaperture images of high electrical pylon on vertical focusing plane at $94 \mathrm{GHz}$ from different viewing angles $\varphi$. Aspect integration is $2.5^{\circ}$. Theoretical resolution is $15 \mathrm{~cm}$ height and $4 \mathrm{~cm}$ length. (a) $\varphi=42^{\circ}$ (b) $\varphi=36^{\circ}$ (c) $\varphi=-216^{\circ}$ (d) $\varphi=191^{\circ}$. The backscattering characteristic is highly sensitive to aspect changes.

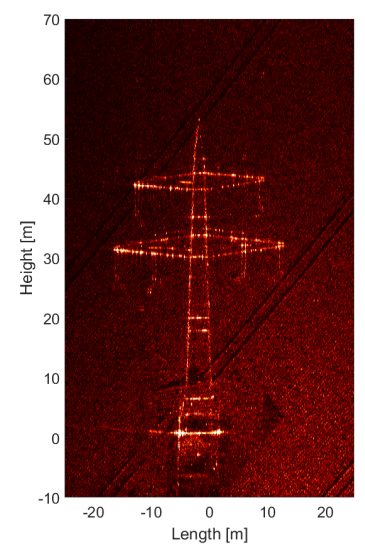

(a)

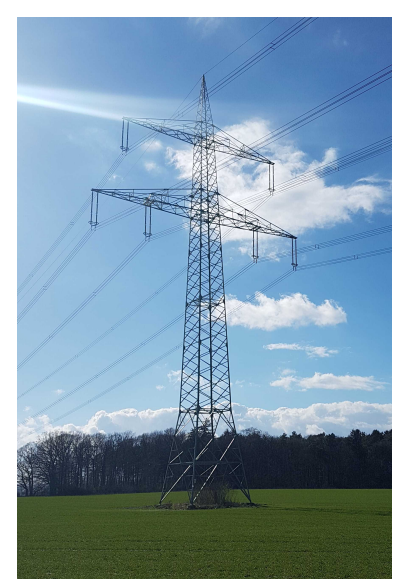

(b)
Figure 11. CSAR target imaging. (a) Estimating object's height from vertical plane (b) Photograph of pylon.

Roberto, 2018. A car-borne SAR system for interferometric measurements: Development status and system enhancements. IEEE International Geoscience and Remote Sensing Symposium, 2018. IGARSS 2018, 6508-6511.

Gehrung, J., Hebel, M., Arens, M., Stilla, U., 2017. An approach to extract moving objects from MLS data using a volumetric background representation. ISPRS Annals of Photogrammetry, Remote Sensing and Spatial Information Sciences, IV-1/W1, 107-114.

Ishimaru, A., Chan, Tsz-King, Kuga, Y., 1998. An imaging technique using confocal circular synthetic aperture radar. IEEE Transactions on Geoscience and Remote Sensing, 36, 1524-1530.

Moreira, A., Prats-Iraola, P., Younis, M., Krieger, G., Hajnsek, I., Papathanassiou, K., 2013. A Tutorial on Synthetic Aperture Radar. IEEE Geoscience and Remote Sensing Magazine, 1, 643.
Palm, S., Sommer, R., Janssen, D., Tessmann, A., Stilla, U., 2019. Airborne Circular W-Band SAR for Multiple Aspect Urban Site Monitoring. IEEE Transactions on Geoscience and Remote Sensing, 1-21, online available: 10.1109/TGRS.2019.2909949.

Palm, S., Sommer, R., Stilla, U., 2018. Mobile Radar Mapping - Subcentimeter SAR Imaging of Roads. IEEE Transactions on Geoscience and Remote Sensing, 56, 6734-6746.

Paparoditis, N., Papelard, J-P., Cannelle, B., Devaux, A., Soheilian, B., David, N., Houzay, E., 2012. Stereopolis II: A multi-purpose and multi-sensor 3D mobile mapping system for street visualisationand 3D metrology. Revue française de photogrammétrie et de télédétection 69-79.

Ponce, O., Prats-Iraola, P., Pinheiro, M., Rodriguez-Cassola, M., Scheiber, R., Reigber, A., Moreira, A., 2014. Fully polarimetric high-resolution 3-D imaging with circular SAR at L-Band. IEEE Transactions on Geoscience and Remote Sensing, 52, 3074-3090.

Reigber, A., Lombardini, F., Viviani, F., Nannini, M., Martinez del Hoyo, A., 2015. Three-dimensional and higher-order imaging with tomographic SAR: Techniques, applications, issues. IEEE International Geoscience and Remote Sensing Symposium, 2015. IGARSS 2015, 2915-2918.

Tessmann, A., Leuther, A., Hurm, V., Massler, H., Wagner, S., Kuri, M., Zink, M., Riessle, M., Stulz, H.-P., Schlechtweg, M., Ambacher, O., 2014. A broadband 220-320 GHz medium power amplifier module. IEEE Compound Semiconductor Integrated Circuit Symposium (CSICs), 2014, 1-4.

Uemoto, J., Nadai, A., Kojima, S., Kobayashi, T., Umehara, T., Matsuoka, T., Uratsuka, S., Satake, M., 2018. Extraction and height estimation of artificial vertical structures based on the wrapped interferometric phase difference within their layovers. ISPRS Journal of Photogrammetry and Remote Sensing, 139, 14 - 29. 